\title{
Thermodynamics of Protein Unfolding-Refolding Transition
}

\author{
A. Anakkar ${ }^{1}$, J. M. Buisine ${ }^{1}$ and J. Jeanfils ${ }^{2 *}$ \\ Université Lille Nord de France, \\ Unité de Dynamique et Structure des Matériaux Moléculaires, EA 4476 \\ ${ }^{1}$ Université de Lille 1, UFR de Physique, 59650 Villeneuve d'Ascq - France \\ ${ }^{2}$ Université du Littoral Côte d'Opale, MREI, 59140 Dunkerque - France \\ Email : joseph.jeanfils@univ-littoral.fr
}

\begin{abstract}
A phenomenological thermodynamic model is developed to investigate, in more detail than usual, the pressuretemperature phase diagram of proteins. Indeed, in the whole of previous studies the specific heat difference $\Delta \mathrm{C}_{\mathrm{P}}$ is treated as pressure and temperature independent. This assumption is wrong, as confirmed by Yamaguchi et al [1] which reported pressure dependence of $\Delta \mathrm{C}_{\mathrm{P}}$ in the case of ribonuclease $\mathrm{A}$. In this work, assuming that the chemical potential has a Taylor series expansion and having an additional thermodynamic piece of information, the analysis shows that it is possible to obtain a complete description of the unfolding-refolding transition of protein, to deduce the volumes, entropies, enthalpies versus transition temperatures and transition pressures and, for the first time, to deduce the specific heat changes versus transition temperatures and transition pressures. Special attention is given to the elliptical shape of the pressure-temperature phase diagram. A quantitative description of the phase transitions in the protein of $\mathrm{Zn}$-Cytochrome $c$ is given.
\end{abstract}

Keywords: Protein folding-unfolding; phase transition; thermodynamic model.

\section{1- Introduction}

The structural organization of proteins is related to their functions in biological systems. Whereas the formation of the amino acid sequences from genetical information is reasonably well known, the process leading to the protein folding is largely unknown. The folding mechanism is related to building of the secondary and tertiary structures of a protein. A suitable protein space conformation is very important when it determines. Spontaneously, a polypeptide chain selects quite rapidly one conformation when a lot of alternative spatial organizations are possible. First of all, atom as well as molecular interaction inside a well-defined protein drives at least for a part the conformational process, in particular the rotation around the peptides bonds linked amino-acids, reaching to $\alpha$-helix or $\beta$-sheet formation. Environmental conditions greatly influence the protein conformational organization. Solvent as well as temperature and pressure can induce conformational changes, leading, in extreme conditions, to the denaturation and/or complete inactivation of the protein. In a first analysis, proteins can exist under two well defined states: native and denatured. Temperature as well as pressure changes affect the molecular organisation of proteins by modifying secondary interactions between amino-acid residues. Increasing or decreasing temperature could lead to a phase change of proteins relating to folding/unfolding process. Environmental conditions influence greatly the thermodynamic properties of a protein rending the analysis the folding/unfolding process very complex. A step by step modelling approach appears as a way to study the protein behaviour during folding/unfolding process. Although this two-state approach is useful to discuss the foldingunfolding process, the transition from native to denatured state must be complicated by including different thermodynamically metastable steps. The transition process from native to denatured state was first thought to be thermodynamically controlled [2, 3], but it is now more certain that the folding - unfolding process is not under the sole control of thermodynamic parameters. Indeed, Levinthal's works [4] indicated that the number of possible spatial configurations of a protein is very high, Wetlaufer [5] assessed at $10^{85}$ seconds the time needed for a one hundred amino-acid peptide to check all conformation possibilities. This is the reason why after thermodynamic considerations, kinetic parameters also have to be considered [5]. Both thermodynamics and kinetic controls drive the protein conformational process [6, 7].

In this study, we are interested in equilibrium states appearing clearly on a phase diagram which are impossible to determine using kinetic effects leading to these equilibrium states.

Phase diagram is a common way of representing the various phases of a substance and the conditions under which each phase exists. It is simply a compact presentation of the known or postulated equation of state of a closed system. However, the protein is an open system that it can exchange matter as well as energy with its surroundings. The equation of state is a relationship between three important properties of pure substances: pressure $\mathrm{P}$, specific volume $\mathrm{V}$, and temperature $\mathrm{T}$. Calculation of phase diagram from volumetric data requires a large computational effort. The equation-of-state- method to reach a complete determination of phase diagram is often unpromising because we usually do not have sufficiently accurate knowledge of the volumetric properties of condensed matter.

Since accurate volumetric data for living matter are missing now, the phase diagram usually used is a graph of 
pressure $\mathrm{P}$ versus temperature $\mathrm{T}$. Lines on the diagram represent conditions $(T, P)$ under which a phase change is at equilibrium. An understanding of such diagrams is essential to thermodynamics, because we will have to refer to a phase diagram in order to obtain the requisite property information. However, few works dealing with the P-T phase diagram of elliptical shape have been found in the case of liquid crystal systems and biological ones. All approaches use the same phenomenological model to describe theoretically the equilibrium curve. They were developed by Hawley [8] for proteins and at the same time by Clarck [9] and Klug and Whalley [10] for liquid crystals and were based on the following assumptions:

- the molar Gibbs free energy difference between the native and denatured states, $\Delta \mathrm{G}=\mathrm{G}_{\mathrm{D}}-\mathrm{G}_{\mathrm{N}}$, is formally the second order Taylor series of $\Delta \mathrm{G}$ expanded with respect to $\mathrm{P}$ and $\mathrm{T}$ around a reference point $\left(\mathrm{P}_{0}, \mathrm{~T}_{0}\right)$,

- since the choice of the reference point is arbitrary it is taken at the centre of the fitted ellipse and at this point the Gibbs free energy difference is maximum,

- the specific heat difference $\Delta C_{P}$ is supposed not to depend on either $\mathrm{P}$ or $\mathrm{T}$.

From a pure mathematical point of view, when truncates the series after the second order term, the thermal expansions $\Delta(\alpha \mathrm{V})$ and the compressibility $\Delta(\chi \mathrm{V})$ differences must be constants, but, under no circumstances, must $\Delta C_{P}$ be so as mentioned by Smeller [11]. If $\Delta C_{P}$ is constant as supposed in all the previous studies $[12,13]$ and since the $\Delta C_{P}=\left(\frac{\partial \Delta H}{\partial T}\right)_{P}$, then the enthalpy must be a linear function of $T$, which is incompatible with the P-T phase diagram of elliptical shape.

Moreover, the Gibbs free energy is given by $\mathrm{dG}=-\mathrm{S}$ $\mathrm{dT}+\mathrm{V} \mathrm{dP}$, then $S=-\left(\frac{\partial G}{\partial T}\right)_{P}$, and since the $C_{P}$ can be also defined by:

$C_{P}=T\left(\frac{\partial S}{\partial T}\right)_{P}$

We can deduce the following expression:

$C_{P}=-T\left(\frac{\partial^{2} G}{\partial T^{2}}\right)_{P}$

From this expression, even if $\Delta \mathrm{G}$ is assumed to be a second order of $\mathrm{P}$ and $\mathrm{T}$, the $\Delta \mathrm{Cp}$ is not a constant. So the possibility $\Delta \mathrm{C}_{\mathrm{P}}$ constant has to be excluded.

The assumption $\Delta C_{P}$ is not constant is confirmed by Yamaguchi et al. [1] who reported pressure dependence of $\Delta C_{P}$ in the case of ribonuclease $A$. Furthermore, even though the reference point is completely arbitrary there is no physical reason to put it at the centre of the ellipse. Finally, considering that the Gibbs free energy difference is maximum at this point implicitly means that energy surfaces $G(T, P)$ of both states have the same maximum. Even if this assumption is attractive it is more difficult to be justified by physical interpretations. That is why another choice, for the more adequate reference point, seems necessary.

Furthermore, for proteins, the thermodynamic system is not a homogeneous one. This is a solution with three phases in equilibrium, both native and denatured phases of the protein and the liquid phase solvent. In other studies, if the effect of solvent is not included in the expression of Gibbs free energy difference, it is not clear why it is neglected and it is not clarified the physical meaning of this absence. In these previous models all thermodynamic properties are not obtained.

Finally, it is well known that for pressure-induced protein unfolding, the volume change $\Delta \mathrm{V}=\mathrm{V}_{\mathrm{D}}-\mathrm{V}_{\mathrm{N}}$, where $V_{D}$ and $V_{N}$ are the volumes of denatured and native states respectively, is an important variable which governs the folding-unfolding equilibrium when pressure is applied. Under some conditions the $\mathrm{V}_{\mathrm{N}}$ could be lower than $\mathrm{V}_{\mathrm{D}}$ and under the others it becomes higher. Then the most important question, which remains open, is how to explain the behaviour change of the volume for each state which is to be consistent with the obtained P-T phase diagram.

The main goals of this work are firstly to clarify the modelling conditions, described in the next section, that are compatible with the experimental data; it is only under these conditions that the principles of thermodynamics can be applied without contradiction unpredictable in the calculations. Secondly, we show that specify the conditions of the modeling is not a disadvantage but rather a means to a better understand the mechanism of the unfoldingrefolding transition of proteins.

The thermodynamic analysis that follows is based on two assumptions about the environment of the protein, a mathematical postulate concerning the chemical potential and a choice of the standard state on the equilibrium curve. We make no assumption about the $\Delta \mathrm{C}_{\mathrm{P}}$. The model must therefore lead to a variation of $\Delta C_{P}$ depending on the temperature and pressure.

For testing ours model, we have used the data obtained from unfolding process of modified $Z n$-Cytochrome $c[12$, 13].

\section{2- The Modelling Conditions}

A heterogeneous closed system is made up of two or more phases where phases considered as an open system within the overall closed system. We now consider the conditions where the heterogeneous system is in a state of internal equilibrium with respect to the three processes of heat transfer, boundary displacement, and mass transfer.

The general result for a closed heterogeneous system consisting of three phases and two components is that, at equilibrium, with respect to processes described above:

$T^{(1)}=T^{(2)}=T^{(3)}$

$P^{(1)}=P^{(2)}=P^{(3)}$

$\mu_{1}^{(1)}=\mu_{1}^{(2)}=\mu_{1}^{(3)}$

$\mu_{2}^{(1)}=\mu_{2}^{(2)}=\mu_{2}^{(3)}$

where the superscript in parentheses denotes the phase and the subscript denotes the component and $\mu$ the chemical potential of components. This set of equation provides the basic criteria for phase equilibrium for our purposes.

The phases considered in this study are the state of water and the two protein's states: native and denatured. In our model, we make two assumptions:

1) the water is under the same thermodynamic conditions of temperature and pressure either inside or outside of the protein,

2) any water molecules alters in any way the state of the protein.

Both assumptions are extremely important because it means that the physicochemical properties are intrinsic to the protein; and that water allowing simply the protein to 
properties revealed when environmental conditions are favorable. With such assumptions, one focus only on studies of protein equilibrium states, i.e. its phase diagram.

To extract all information from the phase diagram we postulate an analytical equation for the chemical potential. In this work on phase-equilibrium thermodynamics one propose to relate the abstract chemical potential of a substance to physical quantities: temperature and pressure. The general regularity observed in the thermodynamic phases' functions allows expanding the chemical potential in Taylor series in $\mathrm{T}$ and $\mathrm{P}$. However, when trying to establish the appropriate relation we need arbitrary reference states, commonly called standard states. Successful application of thermodynamics to real systems is frequently based on a judicious choice of standard states. When we are interested in the thermodynamics of phase equilibrium between two phases, the fundamental equation is the equality of chemical potential; in such a case it is useful to take the standard states on the equilibrium curve, i.e. the standard states for the two phases being the same. As we will see later, this procedure is a powerful way of deducing all thermodynamics properties of a substance as the volumes, entropies, enthalpies and specific heat change along the equilibrium curve.

\section{3- Thermodynamic Analysis of P-T Phase Diagram}

For any phase equilibria between two phases 1 and 2:

$\mu_{1}=\mu_{2}$

where $\mu$ is the chemical potential of phase, with pressure and temperature dependence.

For a one-component two-phase system at equilibrium, relation (1) gives the $P$ and $T$ dependence for the two-phase system $P(T)$ represented by a curve in a PressureTemperature $(P-T)$ phase diagram.

However, we must point out that the analytic relation that links the chemical potential of a pure condensed phase in terms of independent variables $\mathrm{P}$ and $\mathrm{T}$ is unknown.

More or less empirical analytical expressions, in the form of polynomials [14, 15] of exponential [16, 17] or rather often a linear function $[18,19]$, were used to describe the coexistence curves of a pure substance in the P-T phase diagram.

However, no additional information on changes in the volume $\Delta V(T, P)$, entropies $\Delta S(T, P)$, specific heat capacities $\Delta \mathrm{C}_{\mathrm{P}}(T, P)$ or enthalpies $\Delta H(\mathrm{~T}, \mathrm{P})$ associated to the transition is obtained in terms of transition temperatures or pressures.

As the chemical potential is a function $\mu(T, P)$ of the intensive variables $T$ and $P$, we consider that it may be expanded in Taylor series in $\mathrm{T}$ and $\mathrm{P}$ around a reference point $\left(T_{0}, P_{0}\right)$ :

$\mu=\mu_{i 0}+\sum_{i, j} A_{i, j}\left(T-T_{0}\right)^{i}\left(P-P_{0}\right)^{j}$

where the coefficients $A_{\mathrm{ij}}$ are the derivatives of $\mu$ with respect to $P, T$.

In order to relate the abstract chemical potential to physically measurable quantities it is sufficient to truncate the expansions after the quadratic terms. The few data reported in the literature justify no more correction terms (cubic, quartic and so on). Thus, to second order in $T$ and $P$, Eq. (2) becomes:

$$
\begin{aligned}
\mu_{i}(T, P)= & \mu_{i 0}\left(T_{0}, P_{0}\right)-S_{i 0} \Delta T+V_{i 0} \Delta P-\frac{1}{2} \frac{C_{P i 0}}{T_{0}} \Delta T^{2}- \\
& \frac{1}{2} \chi_{i 0} V_{i 0} \Delta P^{2}+\alpha_{i 0} V_{i 0} \Delta T \Delta P
\end{aligned}
$$

where $\mathrm{i}=1,2, \Delta T=T-T_{0}, \Delta P=P-P_{0}$. Here $\mathrm{S}_{\mathrm{i} 0}, \mathrm{C}_{\mathrm{Pi} 0}, \mathrm{~V}_{\mathrm{i} 0}$, $\chi_{\mathrm{i} 0}$ and $\alpha_{\mathrm{i} 0}$ denote entropy, specific heat, volume, isothermal compressibility, and thermal expansivity respectively. It is worth noting that $P$ and $T$ can have all possible values.

The volume and entropy for any $\mathrm{P}$ and $\mathrm{T}$ are obtained by use of Eq. (3):

$V_{i}(T, P)=\left(\frac{\partial \mu_{i}}{\partial P}\right)_{T}$

and

$S_{i}(T, P)=-\left(\frac{\partial \mu_{i}}{\partial T}\right)_{P}$

Since the choice of the reference point is arbitrary we have chosen that the standard states $\mu_{10}\left(T_{0}, P_{0}\right)$ and $\mu_{20}\left(T_{0}, P_{0}\right)$ for the two phases were the same. As indicated above, the choice of a reference point in the equilibrium curve seems adequate.

In this case, the equilibrium relation, Eq.(1), leads to $\Delta(\chi V)_{0} \Delta P^{2}-2 \Delta(\alpha V)_{0} \Delta T \Delta P-2 \Delta V_{0} \Delta P+\frac{\Delta C p_{0}}{T_{0}} \Delta T^{2}+$ $2 \Delta S_{0} \Delta T=0$

where the following notation is used:

$\Delta(\chi V)_{0}=\chi_{20} V_{20}-\chi_{10} V_{10}$

$\Delta(\alpha \mathrm{V})_{0}=\alpha_{20} \mathrm{~V}_{20}-\alpha_{10} \mathrm{~V}_{10}$

$\Delta C_{P 0}=C_{P_{20}}-C_{P_{10}}$

$\Delta V_{0}=V_{20}-V_{10}$

$\Delta S_{0}=S_{20}-S_{10}$

Here, unlike the above expression (Eq.(3)) $\mathrm{P}$ and $\mathrm{T}$ have only the equilibrium values between the two phases. The entropy, enthalpy, volume and specific heat differences between the two phases for any P, T are as follows:

$\Delta S(T, P)=\Delta \mathrm{S}_{0}+\frac{\Delta \mathrm{C}_{\mathrm{P} 0}}{\mathrm{~T}_{0}} \Delta \mathrm{T}-\Delta(\alpha \mathrm{V})_{0} \Delta \mathrm{P}$

$\Delta \mathrm{H}(T, P)=\mathrm{T} \Delta \mathrm{S}(T, P)$

$\Delta V(T, P)=\Delta V_{0}-\Delta(\chi V)_{0} \Delta P+\Delta(\alpha \mathrm{V})_{0} \Delta \mathrm{T}$

$\Delta C_{P}(T, P)=\frac{\Delta C_{P_{0}}}{T_{0}} T$

These equations, Eq.(6a) to Eq.(6e) are the basic ones to describe quantitatively and rigorously coexistence curves of $P-T$ phase diagrams [20].

Since there is no reason to have $\Delta(\chi V)_{0}=0$, it is useful to note that we will only discuss the case of $\Delta(\chi V)_{0} \neq 0$. Then, the Eq. (6a) is the general equation for a conic section and it is interesting to solve it for $\mathrm{T}$ in order to calculate $P-T$ phase diagram i.e. the function $P(T)$ and all thermodynamics proprieties deduced by Eqs.(6b) to (6e).

To determine the explicit forms of the coexistence curves $P(T)$, the entropy, enthalpy, volume and specific 
heat differences for any transition temperature $\mathrm{T}$, we will introduce, in order to simplify the writing of the analytical expressions, new constants $C_{\mathrm{j}}$ which are functions of the initial constants i.e. $T_{0}, P_{0}, \Delta S_{0}, \Delta V_{0}, \Delta C_{\mathrm{Po}}, \Delta(\alpha V)_{0}$ and $\Delta(\chi V)_{0}$.

It is clear from these considerations that the basic equations may be written in the simple forms as follows:

$P=C_{0}+C_{1} T+X \sqrt{C_{3} T^{2}+2 C_{4} T+C_{5}}$

$\Delta V=-\frac{X}{C_{2}} \sqrt{C_{3} T^{2}+2 C_{4} T+C_{5}}$

$\Delta S=-\frac{1}{C_{2}}\left[C_{3} T+C_{4}+X C_{1} \sqrt{C_{3} T^{2}+2 C_{4} T+C_{5}}\right]$

$\Delta C_{P}=\frac{C_{1}^{2}-C_{3}}{C_{2}} \mathrm{~T}$

$\Delta H=T \Delta S$

where $X=+1,-1$ or \pm 1 . It is obvious that if $X=+1$ or $X=-$ 1 , these equations only describe half a conic section. On the other hand $X= \pm 1$ allows the description of the whole conic section. Algebraic rearrangements of the Eq. (6a) reaches to obtain the new constants noted $C_{0}, C_{1}, C_{3}, \mathrm{C}_{4}$ and $C_{5}, C_{2}$ is deduced from Eq.(6e).

From a mathematical point of view, the function $P(T)$ given by Eq.(7a) can either have an elliptic, a parabolic or a hyperbolic shape depending on the sign of

$c_{3}=\frac{1}{\left(\Delta(\chi V)_{0}\right)^{2}}\left[\left(\Delta(\alpha V)_{0}\right)^{2}-\frac{\Delta\left(C_{P}\right)_{0}}{T_{0}} \Delta(\chi V)_{0}\right]$

coefficient, in fact, the curve is:

1. an ellipse if $C_{3}<0$;

2. a parabola or two parallel straight lines if $C_{3}=0$;

3. a hyperbola or two intersecting straight lines if $C_{3}>0$.

The equations established above contain all thermodynamic information about equilibrium state along the coexistence curve if we can know the values of the six coefficients $C_{i}$. If the ones appearing on Eq. (7a) can be deduced from the fitted experimental data, $\mathrm{C}_{2}$ must be determined through any equation from Eqs. (7b) to Eq. (7e) but it requires the knowledge of given thermodynamics data. Indeed, in the case of atmospheric pressure the knowledge of $\Delta V(T)$ or $\Delta H(T)$ (or $\Delta S(T)$ ) or $\Delta C_{P}(T)$ is enough for a complete thermodynamic treatment along the coexistence curve.

\section{4- Application to the $P$ - $T$ Elliptic Phase Diagram of Protein}

The picture which emerged from several experimental data shows a stable native state of the protein in a closed range of the P-T plane. Crossing the boundary of that region, the native conformation loses its stability, and the protein unfolds. Different thermodynamic descriptions of the phase boundary of protein unfolding were developed [8, $11,12,13]$. All these studies show that the shape of the phase diagram of protein unfolding is elliptical. In this case and from Eq. (8), the mathematical constraint ensuring the elliptical shape is

$\rho_{0}^{2}=\frac{T_{0}\left(\Delta(\alpha V)_{0}\right)^{2}}{\Delta\left(C_{P}\right)_{0} \Delta(\chi V)_{0}}<1$
So that the product $\Delta\left(C_{P}\right)_{0} \Delta(\chi V)_{0}$ has to be positive. This implies that both quantities have to have the same sign.

Lesch et al. [13] show that $\rho_{0}^{2}$ is associated to the degree of correlation around a reference point $\left(\mathrm{T}_{0}, \mathrm{P}_{0}\right)$ in the fluctuations of the changes of enthalpy and volume at the denaturing-refolding transition. The expression of Eq. (9) may be used, in general manner, to define a degree of correlations for each transition temperature $\mathrm{T}$ as follows:

$\rho^{2}=\frac{T(\Delta(\alpha V))^{2}}{\Delta\left(C_{P}\right) \Delta(\chi V)}$

According to the approach of the second order Taylor series of chemical potential expanded with respect to $\mathrm{P}$ and $\mathrm{T}$, we deduce

$\Delta(\alpha V)=\frac{C_{1}}{C_{2}}, \Delta(\chi V)=\frac{1}{C_{2}}$ and $\frac{T}{\Delta C_{P}}=\frac{C_{2}}{C_{1}^{2}-C_{3}}$

Then:

$\rho^{2}=\frac{1}{1-\frac{C_{3}}{C_{1}^{2}}}<1$

It seems obvious that this coefficient is positive and inferior to unity since $C_{3}$ is negative. Furthermore, it does not depend on transition temperature. Of course, there is no correlation if $\rho^{2}=0$ and there is full correlations if $\rho^{2}=1$. The last case corresponds to $C_{3}=0$ and it characterizes a parabola curve.

In Figure 1 we show the fit of Eq. (7a) to data reported by Lesch and co-workers for a protein of Zn-Cytochrome $c$ $[12,13]$.

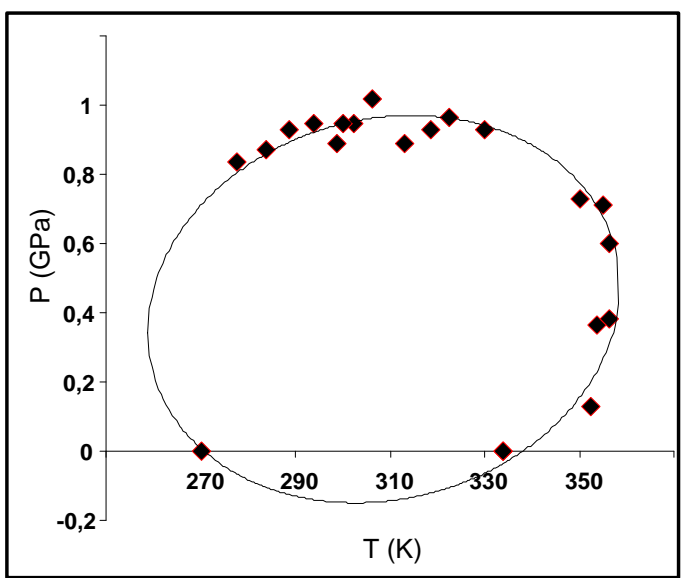

Figure 1. Typical elliptic shape phase diagram. The dots are the experimentally determined points for $\mathrm{Zn}$ Cytochrome c $[12,13]$. The solid line results from Eq. (7a) and parameters as specified in the text.

In order to obtain directly the minimum temperature $T_{1}$ and the maximum temperature $T_{2}$, it is preferable to write the Eq. (7a) as follows

$P=C_{0}+C_{1} T+X \sqrt{C_{3}\left(T-T_{1}\right)\left(T-T_{2}\right)}$

Thereby $C_{4}=C_{3} \frac{T_{1}+T_{2}}{2}$ and $C_{5}=C_{3} T_{1} T_{2}$.

The parameters for this fit are given in Table 1 . The units of measurements used here are the SI units. 
Table 1. Thermodynamic parameters obtained from the fit of ellipse to measure the denaturing-refolding $P-T$ phase boundary in the protein of $\mathrm{Zn}$-Cytochrome c.

\begin{tabular}{ccccc}
\hline Ellipse & \multicolumn{4}{c}{$P=C_{0}+C_{1} T+X \sqrt{C_{3}\left(T-T_{1}\right)\left(T-T_{2}\right)}$} \\
\hline $\mathrm{C}_{0}$ & $\mathrm{C}_{1}$ & $\mathrm{C}_{3}$ & $\mathrm{~T}_{1}$ & $\mathrm{~T}_{2}$ \\
$-210^{-4}$ & $1.3310^{-3}$ & $-1.2410^{-4}$ & 258.8 & 358.6 \\
\hline
\end{tabular}

Since the constants $C_{3}$ and $C_{1}$ are known we can immediately deduce the value of the degree of correlations $\rho^{2}=0.014$. As $\rho^{2}$ is very small, there is almost no correlation between volume and enthalpy changes. This value is very similar to the one computed by Lesch et al. [13]. Then the parameters' values we find are reasonably correct and we can consider the fit of an ellipse to data of $\mathrm{Zn}$-Cytochrome $c$ agrees quite well with the experience, in spite of some errors due to experimental measurements.

As mentioned above, it is necessary to know one value of reference to be able to make a complete thermodynamic study of the coexistence curve. We have taken the value of the specific heat capacities difference $\Delta C_{P}=5.87 \mathrm{~kJ} \mathrm{~mol}^{-1}$ $\mathrm{K}^{-1}$ for $T=333 \mathrm{~K}$ and $P=0.1 \mathrm{MPa}$ [12] which leads to $C_{2}$ $=7.4710^{-3}$ in S.I. units.

The volumes, entropies, enthalpies and specific heat changes along the transition line were then calculated using the Eqs. (7b) to (7c). The results are plotted out in the form of curves in Figures 2 to 9. The first four figures show the variations of these discontinuities according to the transition temperature. The last ones give their evolution according to the transition pressure.

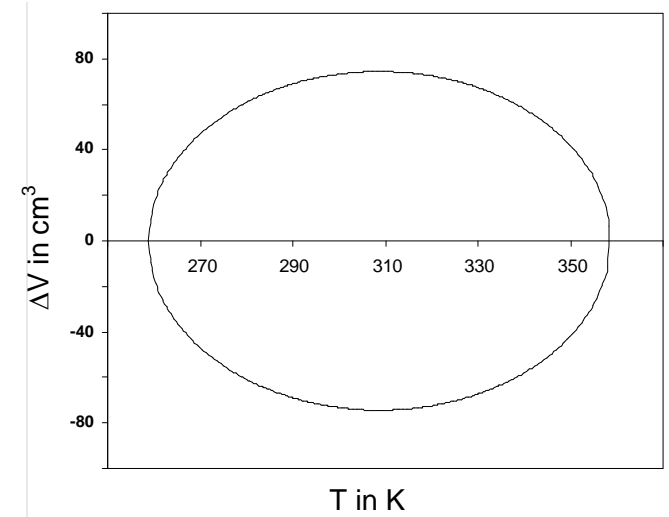

Figure 2. Calculated $\Delta V$ as a function of the transition temperature noted $T: \Delta V=f(T)$.

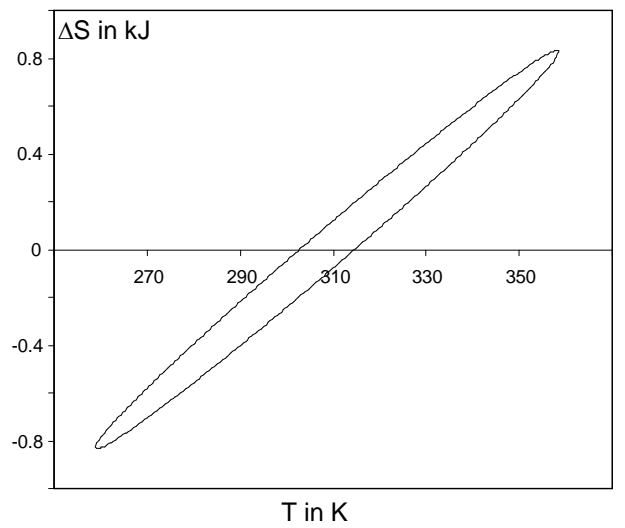

Figure 3. Calculated $\Delta S$ as a function of the transition temperature noted $T: \Delta S=f(T)$.

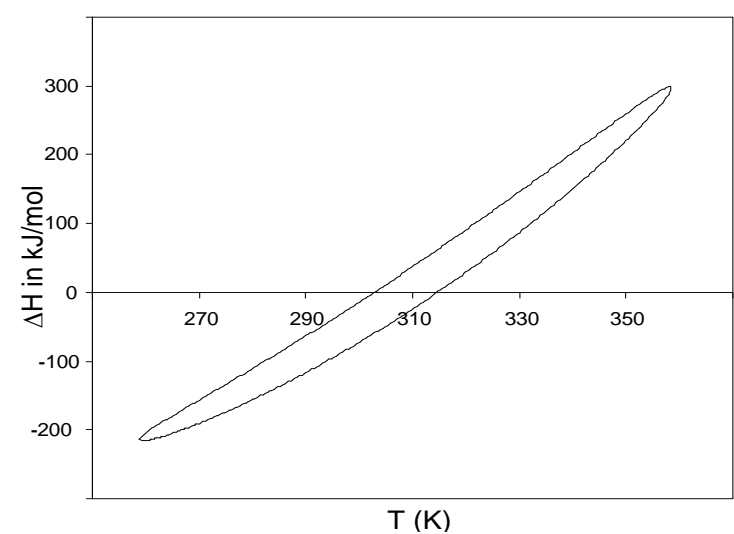

Figure 4. Calculated $\Delta H$ as a function of the transition temperature noted $T: \Delta H=f(T)$.

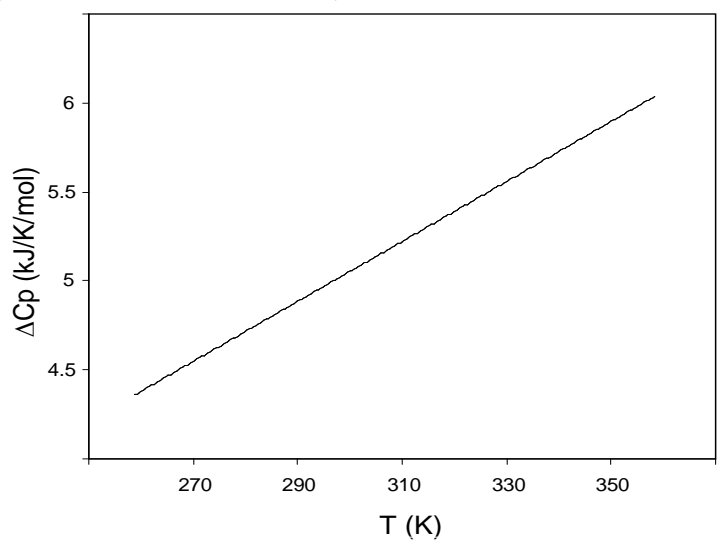

Figure 5. Calculated $\triangle C p$ as a function of the transition temperature noted $T: \Delta C p=f(T)$.

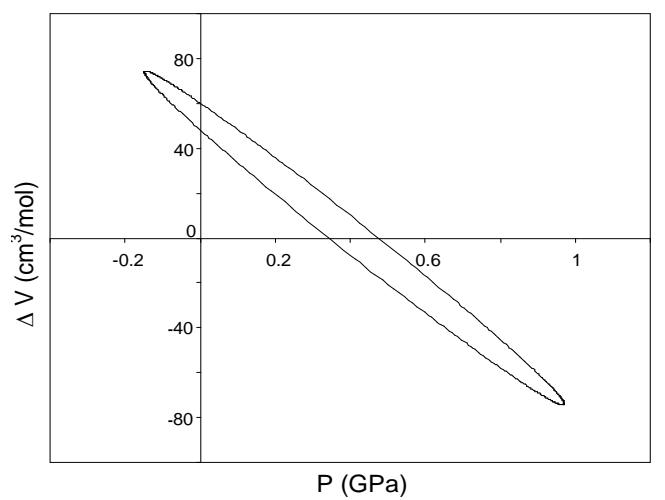

Figure 6. Calculated $\Delta V$ as a function of the transition pressure noted $P: \Delta V=f(P)$.

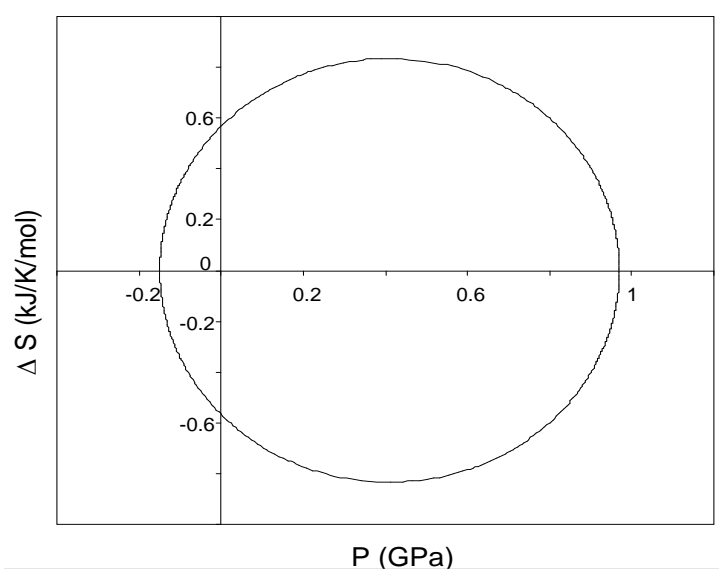

Figure 7. Calculated $\triangle S$ as a function of the transition pressure noted $P: \Delta S=f(P)$. 


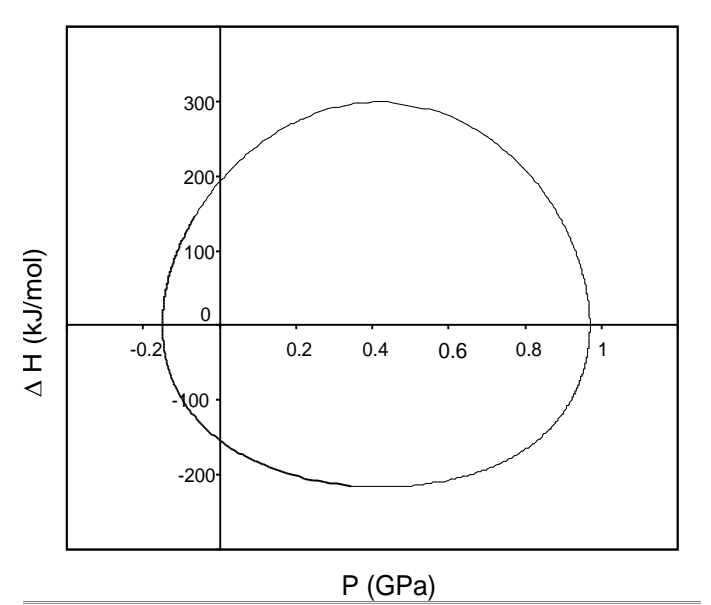

Figure 8. Calculated $\Delta H$ as a function of the transition pressure noted $P: \Delta H=f(P)$.

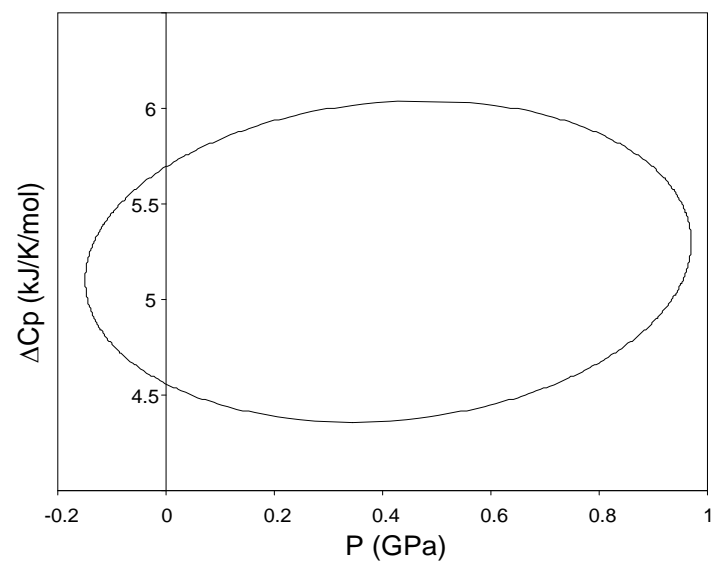

Figure 9. Calculated $\triangle C p$ as a function of the transition pressure noted $P: \Delta C p=f(P)$.

In Table 2 are listed the values deduced from the fit of P-T phase diagram by Lesch et al. [12] and by our model. Our values are very close to those found by the authors mentioned above.

Table 2: Thermodynamics parameters obtained by Lesch et al. $[12,13]$ and by our model for the denatured protein of Zn-cytochrome c

\begin{tabular}{ccc}
\hline Parameters & $\begin{array}{c}\text { From the fit by } \\
\text { Lechs et al. } \\
{[12,13]}\end{array}$ & $\begin{array}{c}\text { From the fit of } \\
\text { our model }\end{array}$ \\
\hline$\Delta \beta\left(\mathrm{cm}^{3} \mathrm{~mol}^{-1} \mathrm{GPa}^{-1}\right)$ & $-148,1$ & $-139,94$ \\
$\Delta \alpha\left(\mathrm{cm}^{3} \mathrm{~mol}^{-1} \mathrm{~K}^{-1}\right)$ & 0,139 & 0,186 \\
$0,91 \mathrm{GPa}^{2} 98 \mathrm{~K}$ & & \\
$\Delta \mathrm{V}\left(\mathrm{cm}^{3} \mathrm{~mol}^{-1}\right)$ & $-74,6$ & $-75,95$ \\
$\Delta \mathrm{S}\left(\mathrm{kJ} \mathrm{mol}^{-1} \mathrm{~K}^{-1}\right)$ & $-0,263$ & $-0,287$ \\
$0,1 \mathrm{MPa} \mathrm{333} \mathrm{K}$ & & \\
$\Delta \mathrm{V}\left(\mathrm{cm}^{3} \mathrm{~mol}^{-1}\right)$ & 65,0 & 67,9 \\
$\Delta \mathrm{S}\left(\mathrm{kJ} \mathrm{mol}^{-1} \mathrm{~K}^{-1}\right)$ & 0,530 & 0,512 \\
\hline
\end{tabular}

\section{5- Discussion}

\section{1- Phase Diagram's Interpretation}

Firstly, since the transition between the native state, $\mathrm{N}$, and the denatured one, $\mathrm{D}$, is characterized by a latent heat, then it is first order and consequently is governed by the Clausius - Clapeyron equation: $\frac{d P}{d T}=\frac{\Delta S}{\Delta V}$

Secondly, the behaviour of each thermodynamics system is governed by Le Chatelier's principle, i.e. when a given system in an equilibrium state is disturbed by the application of an action, a direct reaction occurs in such a way as to diminish the action.

The application of Le Chatelier's principle and the Clausius - Clapeyron equation to the $P-T$ phase diagram leads to its division in four regions as it is shown in Figure 10. The differences between these regions are due to the sign's changes of $\Delta V$ and $\Delta S$ (or $\Delta H=T \Delta S$ ) at transitions. In fact, at constant pressure, if $T$ increases (respectively decreases) then $\Delta H$ is positive (respectively negative) as a consequence of Le Chatelier's principle. When the slope of equilibrium curve is positive (respectively negative), so using the Clausius - Clapeyron equation we deduce that $\Delta \mathrm{H}$ and $\Delta \mathrm{V}$ have the same (respectively opposite) signs. In a similar way, the same results could be deduced when varying pressure at constant temperature (Figure 10).

This interpretation of phase diagram shows that in protein systems, changes in pressure and temperature produce large changes in the phase behaviour. However no information can be deduced from the P-T phase diagram and the Le Chatelier's principle relative to the change of volume of each phase.

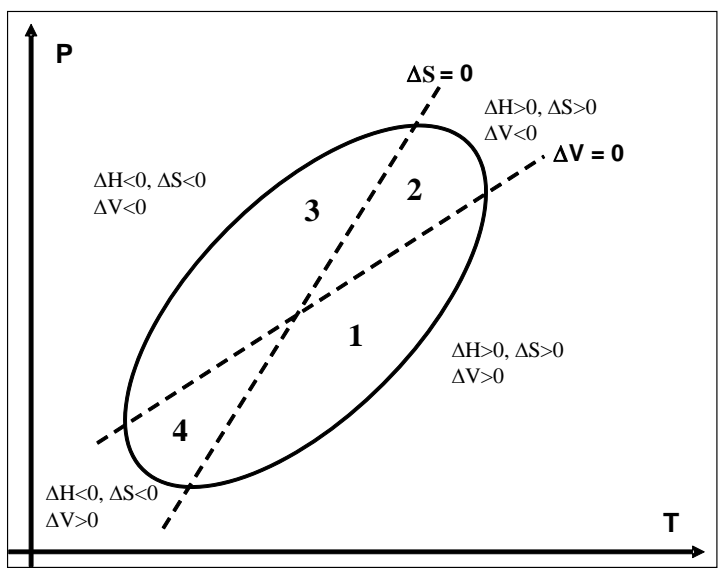

Figure 10. Relative position of the $\Delta S=0$ and $\Delta V=0$ lines compared to the ellipse. This schematic $P-T$ phase diagram shows the four domains which correspond to different signs of entropies and volumes transitions.

\section{2- Volume Behaviours of Each State versus $\boldsymbol{T}$ and $\boldsymbol{P}$}

The variation in $\Delta \mathrm{V}$ influences the folding-unfolding equilibrium according to Le Chatelier's principle, which predicts that a pressure increase (respectively a temperature decrease) favours processes accompanied by negative volume changes and a pressure decrease (respectively a temperature increase) favours processes accompanied by positive volume changes.

Let us consider the effect of heating from the native state, $\mathrm{N}$, to the denatured one, $\mathrm{D}$, at different constant pressures. When the temperature increases, the volume difference $\Delta V=V_{\mathrm{D}^{-}} V_{\mathrm{N}}$ at the transition is positive, then $\mathrm{V}_{\mathrm{N}}$ $<V_{\mathrm{D}}$. But, when the temperature decreases, the volume difference is negative, then $V_{\mathrm{N}}>V_{\mathrm{D}}$. Let us see now what happens with the pressure. We assume that the pressure varies from the $\mathrm{N}$ state to the $\mathrm{D}$ one at different constant temperatures. Thus, when pressure increases one obtains $\mathrm{V}_{\mathrm{N}}$ $>\mathrm{V}_{\mathrm{D}}$, while when it decreases, one gets $\mathrm{V}_{\mathrm{N}}<\mathrm{V}_{\mathrm{D}}$. In order to build-up a physical interpretation of this behaviour we have based our analysis upon the following assumptions: - according to the above equation of the chemical potential, the volume of each phase is considered a linear function of 
$\mathrm{P}$ and $\mathrm{T}$, which is, in first approximation, reasonable in the condensed matter systems,

- at constant temperature if the pressure increases, the volume of the denatured state $\mathrm{V}_{\mathrm{D}}$ must decrease more quickly than that of the native state $\mathrm{V}_{\mathrm{N}}$; it is obvious that it is easier to compress a disordered state than an ordered state,

- with constant pressure if the temperature increases, the volume of the denatured state $\mathrm{V}_{\mathrm{D}}$ must increase more quickly than that of the native state $\mathrm{V}_{\mathrm{N}}$; in fact, it is usually easier to observe the expansion of a disordered state than that of an ordered state.

The schematic representation of the volume change in each state with pressure at fixed temperature and with temperature at fixed pressure is given Figure 11.The results are in qualitative agreement with the experimental P-T phase diagram.
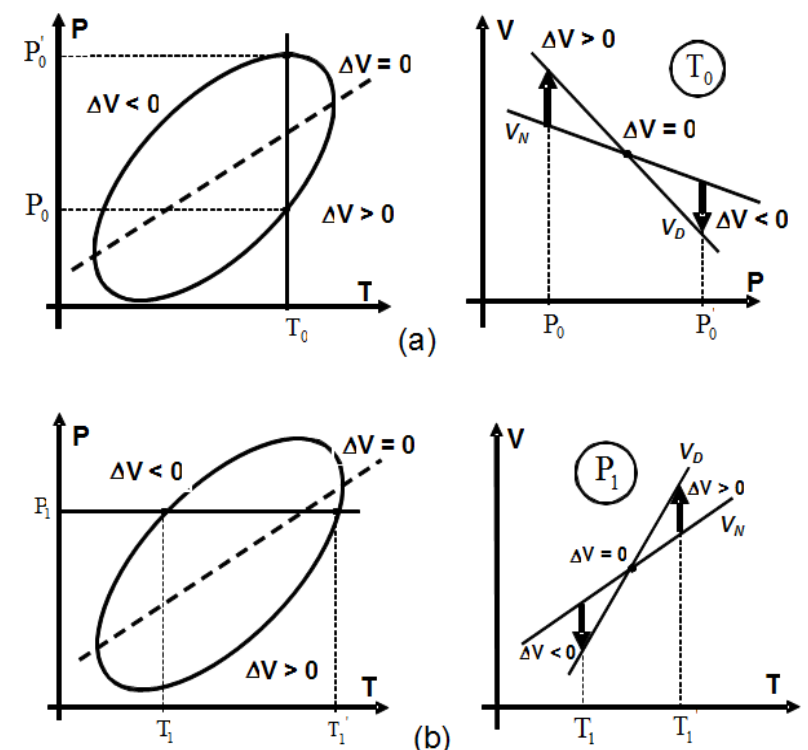

Figure 11: Schematic representation of the volume variation for both states, denatured and native, of protein which is to be consistent with the obtained P-T phase diagram: (a) the volume of each phase is considered as a decrease linear function of $P$ at $T$ constant (example of $T=$ $\left.T_{0}\right)$; (b) the volume of each phase $i$ considered as an increase linear function of $T$ at $P$ constant (example of $P=$ $\left.P_{1}\right)$.

\section{3- Entropy Behaviours of Each State versus $\boldsymbol{T}$ and $\boldsymbol{P}$}

It is well-known that the entropy of a phase is an increasing function of the temperature. However, the two unfolded states are separated by the native state. Then, with fixed pressure, is the entropy of protein in the unfolded state at low temperature lower or higher than that of the unfolded state at high temperature, and similarly by varying pressure at constant temperature. To answer these questions, it is necessary to use the diagram of phase pressure-temperature of protein and the calculated curves (Figures 3 and 5) giving the entropies of transition along this diagram.

The curves describing the behaviour of the entropy of the two states native and denatured protein according to the temperature with constant pressure and according to the pressure at constant temperature are presented in Figure 12.

With constant pressure the entropies of transitions (Figure $12 \mathrm{a}$ ) to the points $\mathrm{A}$ and $\mathrm{B}$ are respectively positive and negative, i.e. $\mathrm{S}_{\mathrm{D}}(\mathrm{A})>\mathrm{S}_{\mathrm{N}}(\mathrm{A})$ and $\mathrm{S}_{\mathrm{D}}(\mathrm{B})<\mathrm{S}_{\mathrm{N}}(\mathrm{B})$; and as $\mathrm{S}_{\mathrm{N}}(\mathrm{A})>\mathrm{S}_{\mathrm{N}}(\mathrm{B})$ then $\mathrm{S}_{\mathrm{D}}(\mathrm{B})<\mathrm{S}_{\mathrm{D}}(\mathrm{A})$ what is fortunately in agreement with the fact that the entropy increases with the temperature.

In order to analyze the variation of the entropy according to the pressure (Figure 12b), it is necessary to specify the value of the temperature. For temperatures $T_{1}$ and $\mathrm{T}_{2}$, the entropy of transition is zero; both temperatures divide the domain of temperature into three intervals:
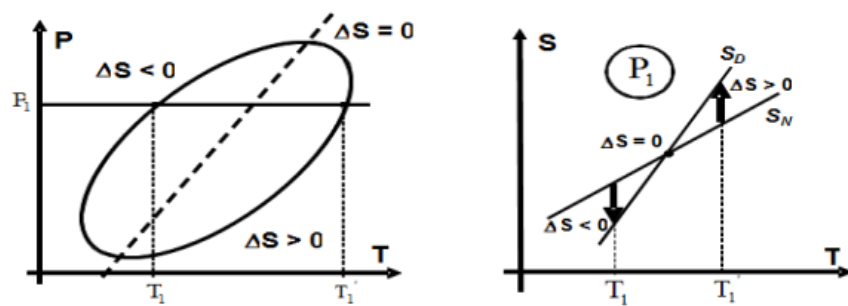

(a)
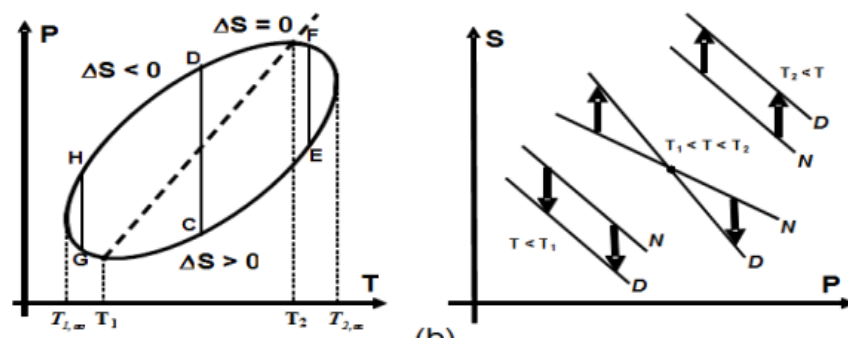

(b)

Figure 12. Schematic representation of the entropy variation for both states, denatured and native, of protein which is to be consistent with the obtained P-T phase diagram: (a) the entropy of each phase is considered as an increase linear function of $T$ at $P$ constant (example of $P=$ $\left.P_{1}\right) ;(b)$ the entropy of each phase is considered as a decrease linear function of $P$ at $T$ constant.

For a constant temperature $T$ between $T_{1}$ and $T_{2}\left(T_{1}<T\right.$ $<T_{2}$ ) the transition entropies at points $\mathrm{C}$ and $\mathrm{D}$ are such as $\mathrm{S}_{\mathrm{D}}(\mathrm{C})-\mathrm{S}_{\mathrm{N}}(\mathrm{C})>0$ and $\mathrm{S}_{\mathrm{D}}(\mathrm{D})-\mathrm{S}_{\mathrm{N}}(\mathrm{D})<0$. As usual, one can suppose that the thermal expansion coefficient $\alpha_{N}$, of protein is positive in the native state; one can deduce, by using the thermodynamic equations from Maxwell, that $\left(\frac{\partial S}{\partial P}\right)_{T}=-\left(\frac{\partial V}{\partial T}\right)_{P}<0$. In other words the entropy is a decreasing function of the pressure. Consequently in the native state we have $S_{\mathrm{N}}(\mathrm{D})<S_{\mathrm{N}}(\mathrm{C})$. By combining this result with the signs of the entropies of transition, we finally deduce that $S_{\mathrm{D}}(\mathrm{D})<S_{\mathrm{D}}(\mathrm{C})$ and reach the conclusion that the thermal expansion coefficient, $\alpha_{D}$, of the denatured state is positive.

If the temperature $T$ is between $T_{2}$ and $T_{2, \infty}$, transition entropies at points $\mathrm{E}$ and $\mathrm{F}$ are both positive. According to Figure 12 they are such as $S_{\mathrm{D}}(\mathrm{F})<S_{\mathrm{D}}(\mathrm{E})$, which enables to deduce that $S_{\mathrm{D}}(\mathrm{F})-S_{\mathrm{D}}(\mathrm{E})<S_{\mathrm{N}}(\mathrm{F})-S_{\mathrm{N}}(\mathrm{E})$. As $S_{\mathrm{N}}(\mathrm{F})<S_{\mathrm{N}}(\mathrm{E})$ and since $\alpha_{\mathrm{N}}>0$, then $S_{\mathrm{D}}(\mathrm{F})<S_{\mathrm{D}}(\mathrm{E})$ and thus $\alpha_{\mathrm{D}}>0$.

- In the last case which corresponds to a temperature range between $T_{1, \infty}$ and $T_{1}$, the transition entropies, for example at points $G$ and $H$, are negative. According to Figure 12-b they are such as $S_{\mathrm{D}}(\mathrm{H})<S_{\mathrm{D}}(\mathrm{G})$, which allows to deduce that $S_{\mathrm{D}}(\mathrm{H})-S_{\mathrm{D}}(\mathrm{G})<S_{\mathrm{N}}(\mathrm{H})-S_{\mathrm{N}}(\mathrm{G})$. As $S_{\mathrm{N}}(\mathrm{H})<$ $S_{\mathrm{N}}(\mathrm{G})$, then $S_{\mathrm{D}}(\mathrm{H})<S_{\mathrm{D}}(\mathrm{G})$ i.e. once again $\alpha_{\mathrm{D}}>0$.

The reasoning carried out above is based on the completely usual assumption that the thermal expansion coefficient of the native state is positive. It is necessary to confirm this sign with an experimental measurement which, 
in the case of proteins, is certainly not easy because the protein is in solution and consequently measurement will contain an additional contribution due to solvent. Finally, the entropy of the denatured phase, which is compared to the diagram of phase Pressure-Temperature on the left of the ellipse, has according to what precedes a smaller value than that which corresponds to the native phase. Thus overall, the low temperature denatured phase is more ordered than the native phase, this last phase being more ordered than the high temperature denatured phase. This result is by no means in contradiction with the principles of thermodynamics since it is necessary to call upon the concept of symmetry which describes the order with long distance. On the other hand, the bond with the structure of the native and denatured states, whether at high or especially at low temperature, has remained an open question so far.

\section{6- Concluding Remarks}

Thermodynamic models have been proposed to analyse the dynamic of cell interaction [21, 22]. This kind of model involves a sophisticated mathematic based on entropy generation reaching to the description of the stationary states in an open system [23].

In our case, we consider the equilibrium sates inside a close system. In this way, a thermodynamic model based on the Taylor series expansion of the chemical potential as a function of temperature and pressure has been extended to investigate the elliptical shape of the pressure-temperature phase diagram of protein in more detail than usual. The analysis carried out above shows that it is possible to determine all thermal properties that characterize the transition between the denatured state and the native one i.e. the volumes, entropies, enthalpies and specific heat changes versus transition temperatures and transition pressures. Reasonable agreement between the experimental data for the Zn-Cytochrome $c$ protein and the calculated values of this model has been found.

In vivo, especially inside cellular membrane, proteins play a fundamental role, either functional or structural. As their environment is changing, proteins have to move their conformational structure. Signal transduction phenomenon is often related to a conformational change reaching to specific cellular activity. In other cases, it is possible that protein activities (i.e enzyme activity) inside biological membranes change local conditions of temperature or pressure inducing so a partial unfolding of protein. Maybe, the unfolding - folding protein process is part of complex regulation pathways driving the control of cell activities. Under these conditions, proteins generate irreversible thermodynamic processes due to various gradients of temperature, pressure and chemical potential. Therefore, a rigorous thermodynamic analysis requires more sophisticated models involving the study of open systems. The method developed by Lucia and Maino [24] based on entropy generation is powerful to study these complex systems by determining their stationary states [22]. The theoretical results of this method can be applied in various fields including the medical one: a mathematical analysis of the dynamics of tumor interaction with the host immune system [21], the human brain [23] or cancer [25].

\section{References}

[1] A. Yamaguchi, T.H. Yamada and K. Akasaka, Thermodynamics of unfolding of ribonuclease A under high pressure. A study by proton NMR, J. Mol. Bio., 250, 689-694, 1995.

[2] C.B Anfinsen. and H.A Schegara, Experimental and theoretical aspects of protein folding, Adv. Prot. Chem., 29, 205-300, 1975.

[3] C. Tanford, Theoretical models for the mechanism of denaturation, Adv. Prot. Chem., 24, 1-95, 1970.

[4] C. Levinthal, Are there pathways for protein folding?, J. Chem. Phys., 65, 44-45, 1968.

[5] D. B. Wetlaufer, Nucleation, rapid folding and globular intrachain regions in proteins, Proc. Natl. Acad. Sci. USA, 70, 697-701, 1973.

[6] C. J. Camacho and D. Thirumalai, Kinetics and Thermodynamics of Folding in Model Proteins. Proc. Natl. Acad. Sci. USA, 90, 6369-6372, 1993.

[7] Z. Guo and D. Thirumalai, Kinetics and thermodynamics of folding of a de-novo designed 4helix Bundle protein. J.Mol. Biol. 263, 323-243, 1996.

[8] S. A. Hawley, Reversible pressure-temperature denaturation of chymotrypsinogen, Biochemistry, 10, 2436-2442, 1971.

[9] N. A. Clark, Thermodynamics of the re-entrant nematic-bilayer smectic A transition, J. Phys., 40, 345-349, 1979.

[10] D. D. Klug and E. Whalley, Elliptic phase boundaries between smectic and nematic phases,. J. Chem. Phys., 71, 1874-877, 1979.

[11] L. Smeller, Pressure-temperature phase diagrams of biomolecules,. Biochimica Biophysica Acta, 1595, 11$29,2002$.

[12] H. Lesch, H. Stadlbauer, J. Friedrich and J.M. Vanderkooi, Stability Diagram and Unfolding of a Modified Cytochrome C: What happens in the transformation regime?, Biophysical Journal, 82, 1644-1653, 2002.

[13] H. Lesch, C. Hecht and J. Friedrich, Protein phase diagrams: The physics behind their elliptic shape, $J$. Chem. Phys., 121, 12671-12675, 2004.

[14] M. Sandmann and A. Würflinger, PVT Measurements on 4'-n-Octyl-Biphenyl-4-Carbonitrile (6CB) and 4'n-Heptyl-Biphenyl-4-Carbonitrile (7CB) up to 300 MPa, Z. Naturforsch., 53a, 233-238, 1998.

[15] W. Spratte and G.M. Schneider, Smectic Polymorphism of Some Bis-(4,4'-nalkoxybenzylidene) - 1,4-phenylenediamines up to 3 kbars by Differential Thermal Analysis, Liq. Cryst., 51, 101-115, 1979.

[16] C. Rein and D. Demus, High pressure differential thermal analysis of nematic compounds with different molecular shapes, Liq. Cryst., 16, 323-331, 1994. 
[17] G. W. H. Höhne and K. Blankenhorn, High pressure DSC investigations on n-alkanes, n-alkane mixtures and polyethylene, Thermochimica Acta, 238, 351-370, 1994.

[18] Y. Maeda, H. Furuya and A. Abe, High pressure differential thermal analysis of dimer liquid crystals: $\alpha, \omega$ Bis [(4,4'-cyanobiphenylyl) oxy] alkanes, Liq. Cryst., 21, 365-371, 1996.

[19] E.A.S Lewis, H.M. Strong and Brown G.H., Volume Measurements and Transitions of MBBA at High Pressure,. Liq. Cryst., 53, 89-99, 1979.

[20] A. Anakkar, Etudes thermodynamiques des phases cristallines liquides. Analyse des points multicritiques et modélisation des diagrammes de phase,. Editions Universitaires Européennes, 2010, ISBN 978-613-152043-3, 2012.
[21] U. Lucia and G. Maino, Thermodynamical analysis of the dynamics of tumor interaction with the host immune system, Physica A, 313, 569-577, 2002.

[22] U. Lucia, Molecular machine as chemicalthermodynamic devices, Chem. Phys. Lett., 556, 242244, 2013.

[23] U. Lucia, Irreversible human brain, Medical hypothesis. 80, 112-114, 2013.

[24] U. Lucia and G. Maino, Maximum or minimum entropy generation for open sytems?, Physica A, 391, 3392-3398, 2012.

[25] U. Lucia, Thermodynamics and cancer stationary states, Physica A, 392, 3648-3653, 2013. 